\title{
Successful Management of Guidewire Kinking in a Patient With Subaortic Septal Bulging Using the Pull-Through Technique During Transapical Transcatheter Aortic Valve Implantation
}

Toshiaki Toyota, MD; Naritatsu Saito, MD; Kenji Minakata, MD; Masao Imai, MD; Kyokun Uehara, MD; Hiroomi Nishio, MD; Yuki Kuroda, MD; Hirotoshi Watanabe, MD;

Tomohiko Taniguchi, MD; Junichi Tazaki, MD; Kazuhiro Yamazaki, MD; Hiroki Daijo, MD; Takeshi Kimura, MD
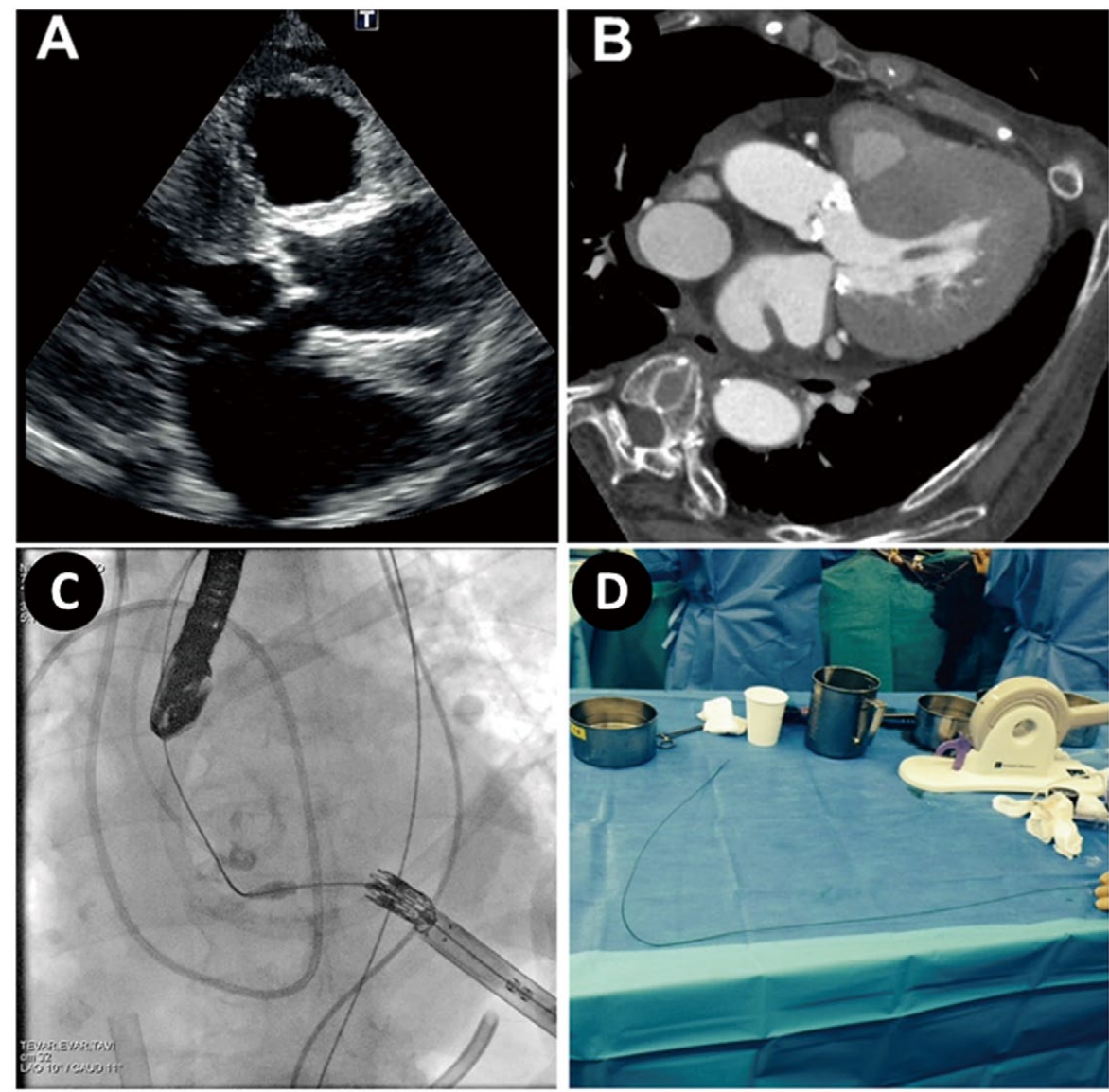

Figure 1. (A) Transthoracic echocardiography, parasternal long-axis view. (B) Reconstructed computed tomography. (C) Fluoroscopy of the kinked guidewire and trapped delivery system. (D) The kinked guidewire after the procedure. The location of the wire kink was consistent with the position of the left ventricular outflow tract.

Received July 24, 2015; accepted October 1, 2015; released online October 23, 2015 Time for primary review: 52 days

Department of Cardiovascular Medicine (T. Toyota, N.S., M.I., H.W., T. Taniguchi, J.T., T.K.), Department of Cardiovascular Surgery (K.M., K.U., H.N., Y.K., K.Y.) and Department of Anesthesiology (H.D.), Graduate School of Medicine, Kyoto University, Kyoto, Japan

Mailing address: Naritatsu Saito, MD, Department of Cardiovascular Medicine, Graduate School of Medicine, Kyoto University, 54 Shogoin

Kawahara-cho, Sakyo-ku, Kyoto 606-8507, Japan. E-mail: naritatu@kuhp.kyoto-u.ac.jp

ISSN-1346-9843 doi:10.1253/circj.CJ-15-0832

All rights are reserved to the Japanese Circulation Society. For permissions, please e-mail: cj@j-circ.or.jp 

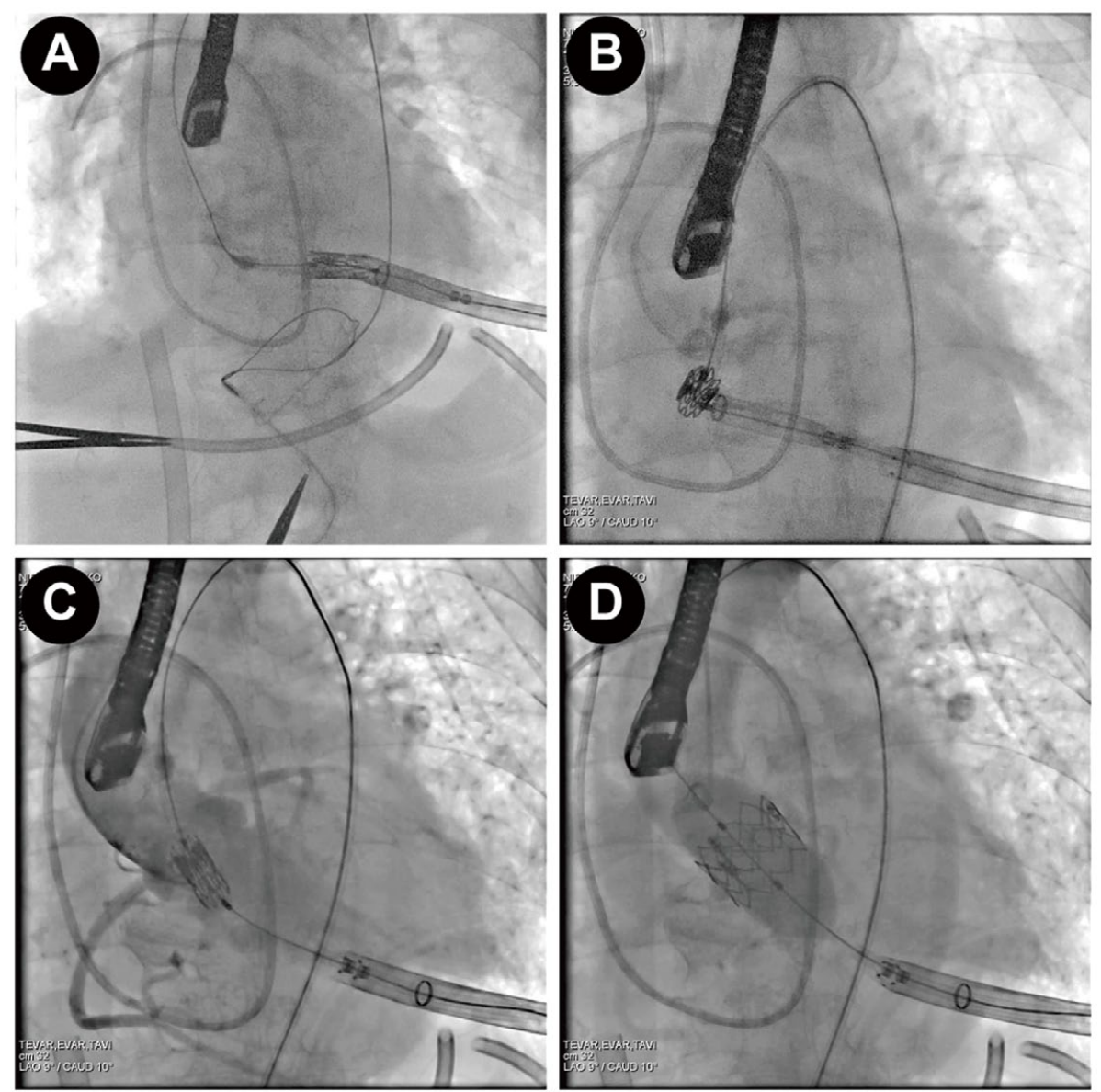

Figure 2. Fluoroscopy during the procedure. (A) The extra-stiff guidewire is caught by the snare catheter. (B) The prosthetic valve is advanced by pushing and pulling both sides of the guidewire. (C) The prosthetic valve is successfully passed through the aortic valve and positioned at the level of the aortic annulus. (D) Successful implantation of the prosthetic valve.

A 90-year-old woman with severe aortic stenosis was scheduled to undergo transcatheter aortic valve implantation (TAVI). Transthoracic echocardiography indicated distinct subaortic septal bulging and severe aortic stenosis (area, $0.34 \mathrm{~cm}^{2}$; peak and mean gradients, 121 and $81 \mathrm{mmHg}$, respectively; ejection fraction, $71 \%$ ), and contrastenhanced computed tomography confirmed marked basal septal bulging and severe calcification in all 3 aortic valves including the commissures (Figures 1A,B). Because of the patient's age and complicated interstitial pneumonia, she was classified as having high risk for surgical aortic valve replacement and was therefore a candidate for TAVI. Due to the tortuousness and narrow diameter of the iliac and femoral artery and the marked basal septal bulging, the transapical approach was chosen.

Extracorporeal membrane oxygenation support was initiated with left femoral artery and right femoral vein cannulation due to hemodynamic instability and pulmonary hypertension after induction of the general anesthesia. After the sixth intercostal thoracotomy, the left ventricular apex was punctured under transesophageal echocardiography guidance and a 24-Fr Ascendra+ introducer sheath (Edwards Lifesciences, Irvine, CA, USA) was inserted into the left ventricle using an Amplatz extra-stiff guidewire (Cook Medical, Bloomington, IN, USA). After pre-dilatation of the aortic valve using a $20-\mathrm{mm}$ balloon, under rapid right ventricular pacing, a 26-mm SAPIEN XT valve (Edwards Lifesciences) was inserted in the left ventricle. The nose cone of the delivery system could not cross the aortic valve because of the steep angle from the left ventricle to the aortic root. After several attempts, the guidewire was kinked, and the tip of the delivery system was trapped under the aortic valve leaflet (Figure 1C; Movie S1). We decided to use the pull-through technique to stabilize the system and to increase crossability. From the right femoral artery, a 7-Fr Judkins-Right (JR) 4.0 guiding catheter was inserted with the 35-cm Amplatz gooseneck snare catheter (Covidien, Plymouth, MN, USA). The tip of the Amplatz extra-stiff guidewire was caught by the snare catheter at the descending aorta. Then, the extra-stiff guidewire was extracted via the right femoral artery sheath. The 7-Fr JR 4.0 guiding catheter was introduced again into the ascending aorta, and the valve delivery system was advanced by pushing and pulling both sides of the guidewire. The prosthetic valve was successfully passed through the aortic valve (Movie S2) and implanted without any complications. Fluoroscopy during the procedure is given in Figure 2. The patient was easily weaned from the extracorporeal membranes oxygenation support, and the operation was completed. Of note, severe kinking of the extra-stiff guidewire was observed after the procedure (Figure 1D). 
The number of high-risk candidates for TAVI is increasing due to the increasing popularity of TAVI. ${ }^{1-5}$ We have herein described the successful management of guidewire kinking in a patient with pronounced subaortic septal bulging, using the pull-through technique during transapical TAVI. The pullthrough technique is a standard technique used in catheter interventions. ${ }^{6,7}$ To the best of our knowledge, however, this is the first report on the use of the pull-through technique for TAVI. Prosthetic valve delivery and stabilization during valve inflation are easily performed using the pull-through technique, which is a simple, safe, and well-established procedure.

None.

\section{Disclosures}

\section{References}

1. Maeda K, Kuratani T, Mizote I, Shimamura K, Takeda Y, Torikai K, et al. Early experiences of transcatheter aortic valve replacement in Japan. Circ J 2013; 77: 359-362.

2. Fuku Y, Goto T, Komiya T, Sakaguchi G, Shimamoto T, Maruo T, et al. Thirty-day outcome of transcatheter aortic valve implantation with the Edwards SAPIEN XT prosthesis via the transiliofemoral approach. Circ J 2014; 78: 1357-1363.
3. Werner N, Sinning JM. Aortic regurgitation after transcatheter aortic valve replacement. Circ J 2014; 78: 811-818.

4. Dvir D, Webb JG. Transcatheter aortic valve-in-valve implantation for patients with degenerative surgical bioprosthetic valves. Circ $J$ 2015; 79: 695-703.

5. Kobayashi J. Changing strategy for aortic stenosis with coronary artery disease by transcatheter aortic valve implantation. Gen Thorac Cardiovasc Surg 2013; 61: 663-668.

6. Matsumoto Y, Nakai K, Tsutsumi M, Iko M, Oishi H, Eto H, et al. A simplified pull-through angioplasty technique without a snare device: A technical note. Interv Neuroradiol 2013; 19: 167-172.

7. Nazarian GK, Myers TV, Bjarnason H, Stackhouse DJ, Dietz CA, Hunter DW. Applications of the Amplatz snare device during interventional radiologic procedures. Am J Roentgenol 1995; 165: 673-678.

\section{Supplementary Files}

Supplementary File 1

Movie S1. Kinking of the guidewire during advancement of the delivery system.

Supplementary File 2

Movie S2. Successful crossing of the aortic valve with the prosthetic valve and delivery system, using the pull-through technique.

Please find supplementary file(s);

http://dx.doi.org/10.1253/circj.CJ-15-0832 